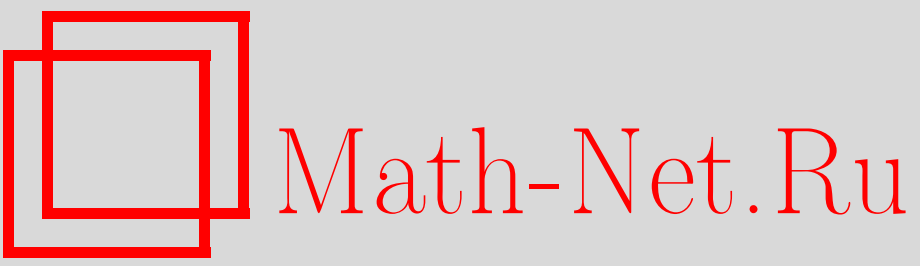

Н. Н. Попов, Л. В. Коваленко, Нелинейная стохастическая задача о растяжении полупространства в условиях ползучести, Вестн. Сам. гос. техн. ун-та. Сер. Физ.-мат. науки, 2007, выпуск 1(), 56-61

DOI: https://doi.org/10.14498/vsgtu488

Использование Общероссийского математического портала Math-Net.Ru подразумевает, что вы прочитали и согласны с пользовательским соглашением

http: //www. mathnet.ru/rus/agreement

Параметры загрузки:

IP : 54.81 .137 .203

26 апреля 2023 г., 16:58:20 


\section{НЕЛИНЕЙНАЯ СТОХАСТИЧЕСКАЯ ЗАДАЧА О РАСТЯЖЕНИИ ПОЛУПРОСТРАНСТВА В УСЛОВИЯХ ПОЛЗУЧЕСТИ}

Рассматривается краевая задача ползучести о растяжении стохастически неоднородного полупространства. Считается, что плоскость, ограничивающая среду, является свободной от напряжений. Задача решена по методу малого параметра в первом приближении. На основе полученного решения проведен статистический анализ поля напряжений на границе среды, исследованы основные особенности краевого эффекта, возникающего в результате стохастических неоднородностей материала. Показано, что вблизи границы полупространства разброс напряжений значительно больше, чем для глубинных слоев. Полученные результаты позволяют адекватно оценить напряженно-деформированное состояние неоднородной среды вблизи ее гранищы.

Приближенный метод расчета трехмерного случайного поля напряжений, возникающего в стохастически неоднородной среде, был дан в [1]. Этот метод основывался на ряде допущений, среди которых одним из существенных было допущение о том, что условия на границе среды пренебрежимо мало влияют на напряженно-деформированное состояние в достаточно удаленных от границы точках. Однако это решение нежелательно применять вблизи свободной поверхности, так как они не обладают достаточной точностью. В связи с изложенным необходимо дополнительное изучение влияния стохастических неоднородностей материала вблизи поверхности, на которой заданы детерминированные граничные условия. Исследование полей напряжений вблизи границы стохастически неоднородной полуплоскости при ползучести было проведено в работах $[2,3]$.

В данной работе рассматривается краевая задача о напряженном состоянии стохастически неоднородного полупространства $x_{3} \geq 0$, находящегося в условиях ползучести. Причем считается, что поверхность $x_{3}=0$ свободна от напряжений, т.е.

$$
\left.\sigma_{i 3}\right|_{x_{3}=0}=0 \quad(i=1,2,3) .
$$

Определяющее соотношение ползучести принимается в соответствии с нелинейной теорией вязкого течения в тензорной форме [1]:

$$
\dot{p}_{i j}=c s^{n-1}\left(\sigma_{i j}-\frac{1}{3} \delta_{i j} \sigma_{k k}\right)\left(1+\alpha U\left(x_{1}, x_{2}, x_{3}\right)\right),
$$

где $s$ - интенсивность напряжений:

$$
s^{2}=\frac{1}{2}\left(3 \sigma_{i j} \sigma_{i j}-\sigma_{i i} \sigma_{j j}\right) .
$$

Здесь и далее $p_{i j}-$ компоненты тензора деформаций ползучести; $\sigma_{i j}-$ компоненты тензора напряжений; $\delta_{i j}-$ символ Кронекера; $\alpha-$ число, характеризующее степень неоднородности материала $(0<\alpha<1) ; c, n-$ постоянные материала. Стохастичность введена в соотношение (2) при помощи случайной однородной функции $U\left(x_{1}, x_{2}, x_{3}\right)$, описывающей флуктуации реологических свойств материала. Математическое ожидание $\langle U\rangle$ и дисперсия $\left\langle U^{2}\right\rangle$ имеют значения 0 и 1 соответственно. Точка означает дифференцирование по времени. По повторяющимся индексам производится суммирование от 1 до 3.

Компоненты тензора напряжений $\sigma_{i j}$ удовлетворяют уравнениям равновесия

$$
\sigma_{i j, j}=0,
$$

а компоненты тензора скоростей деформаций $\dot{p}_{i j}-$ условиям

$$
\Lambda_{i j k} \Lambda_{l m n} \dot{p}_{k m, i j}=0 \text {, }
$$

которые получены из уравнений совместности деформаций путем дифференцирования по времени. Здесь $\Lambda_{i j k}$ - единичный антисимметричный псевдотензор.

Соотношения (2)-(4) при краевых условиях (1) задают стохастическую задачу ползучести, которая в дальнейшем решается приближенно относительно напряжений.

Тензор напряжений $\sigma_{i j}$ с учетом (1) может быть представлен в виде суммы двух слагаемых 


$$
\sigma_{i j}=\sigma_{i j}^{0}+\sigma_{i j}^{*}, \quad\left\langle\sigma_{i j}\right\rangle=\sigma_{i j}^{0}, \quad\left\langle\sigma_{i j}^{*}\right\rangle=0,
$$

где $\sigma_{i j}^{0}$ - детерминированная составляющая, а $\sigma_{i j}^{*}$ - флуктуации напряжений.

Линеаризация задачи ползучести (2)-(4) была произведена в работе [1]. С целью физической линеаризации функция $s^{n-1}$ была разложена в степенной ряд и в этом разложении были учтены только линейные члены. Для статистической линеаризации определяющего соотношения (2) использовалось корреляционное приближение теории случайных функций, т.е. предполагалось, что произведениями вида $\sigma_{i j}^{*} \sigma_{k l}^{*}, \alpha U \sigma_{i j}^{*}$ допустимо пренебречь. В результате система (1)-(4) сводится к системе дифференциальных уравнений в частных производных относительно флуктуаций напряжений $\sigma_{i j}^{*}$ :

$$
\begin{gathered}
\sigma_{i j, j}^{*}=0, \\
\sigma_{i i, 12}^{*}\left(k_{3} l_{i}-1\right)+\alpha U_{, 12} l_{3}=3\left(\sigma_{13,23}^{*}+\sigma_{23,13}^{*}-\sigma_{12,33}^{*}-\sigma_{33,12}^{*}\right), \\
\sigma_{i i, 13}^{*}\left(k_{2} l_{i}-1\right)+\alpha U_{, 13} l_{2}=3\left(\sigma_{23,12}^{*}+\sigma_{12,23}^{*}-\sigma_{13,22}^{*}-\sigma_{22,13}^{*}\right), \\
\sigma_{i i, 23}^{*}\left(k_{1} l_{i}-1\right)+\alpha U_{, 22} l_{1}=3\left(\sigma_{12,13}^{*}+\sigma_{13,12}^{*}-\sigma_{23,11}^{*}-\sigma_{11,23}^{*}\right), \\
\sigma_{i i, 11}^{*}\left(k_{2} l_{i}-1\right)+\sigma_{i i, 22}^{*}\left(k_{1} l_{i}-1\right)+3\left(\sigma_{11,22}^{*}+\sigma_{22,11}^{*}\right)+\alpha\left(U_{, 22} l_{1}+U_{, 11} l_{2}\right)=6 \sigma_{12,12}^{*}, \\
\sigma_{i i, 11}^{*}\left(k_{3} l_{i}-1\right)+\sigma_{i i, 33}^{*}\left(k_{1} l_{i}-1\right)+3\left(\sigma_{11,33}^{*}+\sigma_{33,11}^{*}\right)+\alpha\left(U_{, 33} l_{1}+U_{, 11} l_{3}\right)=6 \sigma_{13,13}^{*}, \\
\sigma_{i i, 22}^{*}\left(k_{3} l_{i}-1\right)+\sigma_{i i, 33}^{*}\left(k_{2} l_{i}-1\right)+3\left(\sigma_{22,33}^{*}+\sigma_{33,22}^{*}\right)+\alpha\left(U_{, 33} l_{2}+U_{, 22} l_{3}\right)=6 \sigma_{23,23}^{*},
\end{gathered}
$$

где

$$
\begin{gathered}
l_{1}=3 \sigma_{11}^{0}-\sigma_{i i}^{0}, \quad l_{2}=3 \sigma_{22}^{0}-\sigma_{i i}^{0}, \quad l_{3}=3 \sigma_{33}^{0}-\sigma_{i i}^{0}, \quad k_{i}=\frac{(n-1) l_{i}}{2 s_{0}^{2}}, \\
s_{0}^{2}=\left(\sigma_{11}^{0}\right)^{2}+\left(\sigma_{22}^{0}\right)^{2}+\left(\sigma_{33}^{0}\right)^{2}-\sigma_{11}^{0} \sigma_{22}^{0}-\sigma_{11}^{0} \sigma_{33}^{0}-\sigma_{22}^{0} \sigma_{33}^{0} .
\end{gathered}
$$

Система (6) состоит из девяти уравнений, но линейно независимыми из них является только шесть первых уравнений.

Краевые условия для системы (6) с учетом (1),(5) имеют вид

$$
\left.\sigma_{i 3}^{*}\right|_{x_{3}=0}=0 \text {. }
$$

Пусть однородное случайное поле $U\left(x_{1}, x_{2}, x_{3}\right)$, описывающее возмущения механических свойств материала, допускает представление в виде ряда [4]:

$$
U\left(x_{1}, x_{2}, x_{3}\right)=\sum_{k=1}^{\infty} A^{(k)} \cos \left(\omega \beta_{s}^{(k)} x_{s}+\varphi^{(k)}\right),
$$

где $\omega$ - большой параметр, имеющий размерность, обратную длине; $\beta_{s}^{(k)}$ - безразмерные величины порядка единицы; $A^{(k)}$ - центрированные случайные величины; $\varphi^{(k)}$ - случайные величины, распределенные равномерно на участке $(0,2 \pi)$, причем все случайные величины $A^{(k)}, \varphi^{(k)}$ независимы.

Для удобства выкладок целесообразно перейти к комплексным функциям

$$
\tilde{U}=\sum_{k=1}^{\infty} \tilde{A}^{(k)} \exp \left(i \omega \beta_{S}^{(k)} x_{S}\right)
$$

где $\tilde{A}^{(k)}=A^{(k)} \exp \left(i \varphi^{(k)}\right)$.

Решение системы уравнений (6), (7) можно представить в виде

$$
\sigma_{i j}^{*}=\sum_{k=1}^{\infty}\left(v_{i j}^{(k)}+w_{i j}^{(k)}\right),
$$

где $v_{i j}{ }^{(k)}$ - частное решение системы (6), полученное при замене функции $\widetilde{U} k$-тым членом разложения (9), а $w_{i j}{ }^{(k)}$ - решение соответствующей (6) однородной системы.

В дальнейшем, где это не приводит к недоразумениям, верхний индекс $k$ будет опускаться. 
Функции $v_{i j}$ будем искать в виде

$$
v_{i j}=f_{i j} \exp \left(i \omega \beta_{s} x_{s}\right), \quad f_{i j}=\text { const . }
$$

После подстановки (11) в (6) для нахождения $f_{i j}$ получается система алгебраических уравнений вида:

$$
\left\{\begin{array}{l}
f_{11} \beta_{1}+f_{12} \beta_{2}+f_{13} \beta_{3}=0, \\
f_{12} \beta_{1}+f_{22} \beta_{2}+f_{23} \beta_{3}=0, \\
f_{13} \beta_{1}+f_{23} \beta_{2}+f_{33} \beta_{3}=0, \\
f_{i i} \beta_{1} \beta_{2}\left(k_{3} l_{i}-1\right)+\alpha \tilde{A} \beta_{1} \beta_{2} l_{3}=3\left(f_{13} \beta_{2} \beta_{3}+f_{23} \beta_{1} \beta_{3}-f_{12} \beta_{3}^{2}-f_{33} \beta_{1} \beta_{2}\right), \\
f_{i i} \beta_{1} \beta_{3}\left(k_{2} l_{i}-1\right)+\alpha \tilde{A} \beta_{1} \beta_{3} l_{2}=3\left(f_{23} \beta_{1} \beta_{2}+f_{12} \beta_{2} \beta_{3}-f_{23} \beta_{1}^{2}-f_{22} \beta_{2} \beta_{3}\right), \\
f_{i i} \beta_{2} \beta_{3}\left(k_{1} l_{i}-1\right)+\alpha \tilde{A} \beta_{2} \beta_{3} l_{1}=3\left(f_{12} \beta_{1} \beta_{3}+f_{13} \beta_{1} \beta_{2}-f_{23} \beta_{1}^{2}-f_{11} \beta_{2} \beta_{3}\right) .
\end{array}\right.
$$

Ряд $\sum_{k=1}^{\infty} v_{i j}{ }^{(k)}$ задает решение задачи вдали от границы полупространства без учета краевого эффекта.

Функции $w_{i j}$ имеют характер пограничного слоя: они быстро затухают по мере удаления от плоскости $x_{3}=0$. Их можно искать в виде

$$
w_{i j}=\varphi_{i j}\left(x_{3}\right) \exp \left(i \omega\left(\beta_{1} x_{1}+\beta_{2} x_{2}\right)\right),
$$

где функции $\varphi_{i j}\left(x_{3}\right)$ удовлетворяют системе дифференциальных уравнений:

$$
\left\{\begin{array}{l}
i \omega \beta_{1} \varphi_{11}+i \omega \beta_{2} \varphi_{12}+\varphi_{13,3}=0, \\
i \omega \beta_{1} \varphi_{12}+i \omega \beta_{2} \varphi_{22}+\varphi_{23,3}=0 \\
i \omega \beta_{1} \varphi_{13}+i \omega \beta_{2} \varphi_{23}+\varphi_{33,3}=0, \\
-\omega^{2} \beta_{1} \beta_{2}\left[\varphi_{11}\left(k_{3} l_{1}-1\right)+\varphi_{22}\left(k_{3} l_{2}-1\right)+\varphi_{33}\left(k_{3} l_{3}+2\right)\right]=3\left[i \omega \beta_{2} \varphi_{13,3}+i \omega \beta_{1} \varphi_{23,3}-\varphi_{12,33}\right], \\
i \omega \beta_{1}\left[\varphi_{11,3}\left(k_{2} l_{1}-1\right)+\varphi_{22,3}\left(k_{2} l_{2}+2\right)+\varphi_{33,3}\left(k_{2} l_{3}-1\right)\right]=3\left[i \omega \beta_{2} \varphi_{12,3}-\omega^{2} \beta_{1} \beta_{2} \varphi_{23}+\omega^{2} \beta_{2}^{2} \varphi_{13}\right], \\
i \omega \beta_{2}\left[\varphi_{11,3}\left(k_{1} l_{1}+2\right)+\varphi_{22,3}\left(k_{1} l_{2}-1\right)+\varphi_{33,3}\left(k_{1} l_{3}-1\right)\right]=3\left[i \omega \beta_{1} \varphi_{12,3}-\omega^{2} \beta_{1} \beta_{2} \varphi_{13}+\omega^{2} \beta_{1}^{2} \varphi_{23}\right] .
\end{array}\right.
$$

Эта система получена подстановкой представления (13) в однородную систему, соответствующую (6).

Все дальнейшие выкладки выполнены для случая, когда растяжение полупространства в направлении осей $x_{1}$ и $x_{2}$ производится равными постоянными напряжениями:

$$
\sigma_{11}^{0}=\sigma_{22}^{0}=\sigma^{0}, \quad \sigma_{33}^{0}=0 .
$$

Решая систему (12), находим константы $f_{i j}$ :

$$
\begin{array}{ll}
f_{i 3}=-\frac{\alpha \sigma^{0}\left(\beta_{i} \beta_{3}-\delta_{i 3} \beta^{2}\right)\left(\beta_{1}^{2}+\beta_{2}^{2}-\beta_{3}^{2}\right)}{\Delta} & (i=1,2,3), \\
f_{i j}=-\frac{\alpha \sigma^{0}\left(\beta_{i} \beta_{j}-\delta_{i j} \beta^{2}\right) 2 \beta_{3}^{2}+\delta_{i j}\left(\beta_{1}^{2}+\beta_{2}^{2}-\beta_{3}^{2}\right)^{2}}{\Delta} & (i, j=1,2),
\end{array}
$$

где

$$
\beta^{2}=\beta_{1}^{2}+\beta_{2}^{2}+\beta_{3}^{2}, \quad \Delta=n\left(\beta_{1}^{2}+\beta_{2}^{2}-\beta_{3}^{2}\right)^{2}+(3+n) \beta_{3}^{2}\left(\beta_{1}^{2}+\beta_{2}^{2}\right) .
$$

Решение системы для функций $\varphi_{i j}\left(x_{3}\right)$ будем искать в виде

$$
\varphi_{i j}=\sum_{s=1}^{7} C_{s} X_{i j}^{(s)} \mathrm{e}^{r_{x} x_{3}} .
$$

Здесь $C_{s}$ - произвольные постоянные, $X_{i j}^{(s)}$ - координаты собственного вектора, соответствующего корню $r_{s}$ характеристического уравнения системы для $\varphi_{i j}\left(x_{3}\right)$, которое имеет вид 


$$
\operatorname{det} B(r)=\left|\begin{array}{cccccc}
i \omega \beta_{1} & 0 & 0 & i \omega \beta_{2} & r & 0 \\
0 & i \omega \beta_{2} & 0 & i \omega \beta_{1} & 0 & r \\
0 & 0 & r & 0 & i \omega \beta_{1} & i \omega \beta_{2} \\
\omega^{2} \beta_{1} \beta_{2} Q_{31} & \omega^{2} \beta_{1} \beta_{2} Q_{32} & \omega^{2} \beta_{1} \beta_{2} Q_{33} & -3 r^{2} & 3 i \omega \beta_{2} r & 3 i \omega \beta_{1} r \\
i \omega \beta_{1} r Q_{21} & i \omega \beta_{1} r Q_{22} & i \omega \beta_{1} r Q_{23} & -3 i \omega \beta_{2} r & -3 \omega^{2} \beta_{2}^{2} & 3 \omega^{2} \beta_{1} \beta_{2} \\
i \omega \beta_{2} r Q_{11} & i \omega \beta_{2} r Q_{12} & i \omega \beta_{2} r Q_{13} & -3 i \omega \beta_{1} r & 3 \omega^{2} \beta_{1} \beta_{2} & -3 \omega^{2} \beta_{1}^{2}
\end{array}\right|=0
$$

где

$$
Q_{i j}=3 \delta_{i j}-1+k_{i} l_{j} .
$$

Раскрывая определитель (17), преобразуем характеристическое уравнение к виду:

$$
r\left(r^{2}-\omega^{2} \beta_{1}^{2}-\omega^{2} \beta_{2}^{2}\right)\left[(3+n) \omega^{2}\left(\beta_{1}^{2}+\beta_{2}^{2}\right) r^{2}-n\left(r^{2}+\omega^{2} \beta_{1}^{2}+\omega^{2} \beta_{2}^{2}\right)^{2}\right]=0 .
$$

Корни этого уравнения при $n \neq 1$ определяются соотношениями

$$
r_{1}=0, \quad r_{2,3}= \pm \omega \sqrt{\beta_{1}^{2}+\beta_{2}^{2}}, \quad r_{4,5}= \pm \omega(a+i b), \quad r_{6,7}= \pm \omega(a-i b),
$$

где

$$
a=\sqrt{\beta_{1}^{2}+\beta_{2}^{2}} \frac{\sqrt{3+n}}{2 \sqrt{n}}, \quad b=\sqrt{\beta_{1}^{2}+\beta_{2}^{2}} \frac{\sqrt{3(n-1)}}{2 \sqrt{n}} .
$$

При $n=1$ корни характеристического уравнения (18) являются кратными и общее решение системы для $\varphi_{i j}\left(x_{3}\right)$ будет иметь другой вид. Этот случай здесь не рассматривается.

Координаты собственных векторов $X_{i j}^{(s)}$ определяются из уравнения $B(r) \cdot X=0$. Для $r_{1}$ они имеют значения:

$$
X_{11}^{1}=-2 \omega^{2} \beta_{2}^{2}, \quad X_{22}^{1}=-2 \omega^{2} \beta_{1}^{2}, \quad X_{33}^{1}=-\omega^{2}\left(\beta_{1}^{2}+\beta_{2}^{2}\right), \quad X_{12}^{1}=\omega^{2} \beta_{1} \beta_{2}, \quad X_{13}^{1}=X_{23}^{1}=0 ;
$$

для $r_{2}, r_{3}$ :

$$
\begin{gathered}
X_{11}^{s}=-X_{22}^{s}=-2 \omega^{2} \beta_{1} \beta_{2}, \quad X_{33}^{s}=0, \\
X_{12}^{s}=-\omega^{2}\left(\beta_{2}^{2}-\beta_{1}^{2}\right), \quad X_{13}^{s}=i r_{s} \omega \beta_{2}, \quad X_{23}^{s}=-i r_{s} \omega \beta_{2} \quad(s=2,3) ;
\end{gathered}
$$

для $r_{4}-r_{7}$ :

$$
\begin{gathered}
X_{11}^{s}=-i r_{s}^{2}\left(\omega^{2} \beta_{2}^{2}-\omega^{2} \beta_{1}^{2}-r_{s}^{2}\right), \quad X_{22}^{s}=-i r_{s}^{2}\left(\omega^{2} \beta_{1}^{2}-\omega^{2} \beta_{2}^{2}-r_{s}^{2}\right), \\
X_{33}^{s}=-i \omega^{2}\left(\beta_{1}^{2}+\beta_{2}^{2}\right)\left(\omega^{2} \beta_{1}^{2}+\omega^{2} \beta_{2}^{2}+r_{s}^{2}\right), \quad X_{12}^{s}=2 i r_{s}^{2} \omega^{2} \beta_{1} \beta_{2}, \\
X_{13}^{s}=r_{s} \omega \beta_{1}\left(\omega^{2} \beta_{1}^{2}+\omega^{2} \beta_{2}^{2}+r_{s}^{2}\right), \quad X_{23}^{s}=r_{s} \omega \beta_{2}\left(\omega^{2} \beta_{1}^{2}+\omega^{2} \beta_{2}^{2}+r_{s}^{2}\right) \quad(s=\overline{4,7}) .
\end{gathered}
$$

Таким образом, общее решение системы (6) получается подстановкой (11), (13), (15), (16) и (18) в выражение (10).

Определим теперь неизвестные константы $C_{s}$. При быстрой осцилляции случайного поля микронеоднородностей краевой эффект должен быстро затухать по мере удаления от границы полупространства. В силу этого решения (10) при $x_{3} \rightarrow \infty$ должно быть ограниченным и совпадать со стационарным, полученным в работе [1]. Корни характеристического уравнения $r_{2}, r_{4}$ и $r_{6}$ имеют положительные действительные части и поэтому в силу условия ограниченности решения $C_{2}=C_{4}=C_{6}=0$. Совпадение полученного решения со стационарным гарантируется условием $C_{1}=0$. Остальные три константы могут быть найдены из краевых условий

используя которые, получим

$$
\left.\varphi_{i 3}\right|_{x_{3}=0}=-\left.f_{i 3}\right|_{x_{3}=0},
$$

$$
\begin{gathered}
C_{3}=0, \quad C_{5}=\alpha \sigma^{0} \frac{\left(\beta_{1}^{2}+\beta_{2}^{2}-\beta_{3}^{2}\right)\left(\omega \beta_{3}+i r_{7}\right)}{\Delta \omega^{2}\left(\omega^{2} \beta_{1}^{2}+\omega^{2} \beta_{2}^{2}+r_{5}^{2}\right)\left(r_{5}-r_{7}\right)}, \\
C_{7}=\alpha \sigma^{0} \frac{\left(\beta_{1}^{2}+\beta_{2}^{2}-\beta_{3}^{2}\right)\left(\omega \beta_{3}+i r_{5}\right)}{\Delta \omega^{2}\left(\omega^{2} \beta_{1}^{2}+\omega^{2} \beta_{2}^{2}+r_{7}^{2}\right)\left(r_{7}-r_{5}\right)} .
\end{gathered}
$$

После подстановки найденных констант в (10), решение задачи примет вид: 


$$
\begin{aligned}
& \sigma_{11}^{*}=\sum_{k=0}^{\infty} \frac{\alpha \sigma^{0}}{\Delta} \exp \left(i \omega\left(\beta_{1}^{(k)} x_{1}+\beta_{2}^{(k)} x_{2}\right)\right)\left\{-\left[2\left(\beta_{3}^{(k)}\right)^{2}\left(\left(\beta_{1}^{(k)}\right)^{2}-\left(\beta^{(k)}\right)^{2}\right)+\right.\right. \\
& \left.+\left(\left(\beta_{1}^{(k)}\right)^{2}+\left(\beta_{2}^{(k)}\right)^{2}-\left(\beta_{3}^{(k)}\right)^{2}\right)^{2}\right] \exp \left(i \omega \beta_{3}^{(k)} x_{3}\right)-\frac{\left(\left(\beta_{1}^{(k)}\right)^{2}+\left(\beta_{2}^{(k)}\right)^{2}-\left(\beta_{3}^{(k)}\right)^{2}\right) i}{r_{5}^{(k)}-r_{7}^{(k)}} \times \\
& \times\left[\frac{\left(\omega \beta_{3}^{(k)}+i r_{7}^{(k)}\right) r_{5}^{(k) 2}}{\omega^{2}\left(\omega^{2} \beta_{1}^{(k) 2}+\omega^{2} \beta_{2}^{(k) 2}+r_{5}^{(k) 2}\right)}\left(\omega^{2} \beta_{2}^{(k) 2}-\omega^{2} \beta_{1}^{(k) 2}-r_{5}^{(k) 2}\right) \mathrm{e}^{r_{5}^{(k)} x_{3}}-\right. \\
& \left.\left.-\frac{\left(\omega \beta_{3}^{(k)}+i r_{5}^{(k)}\right) r_{7}^{(k) 2}}{\omega^{2}\left(\omega^{2} \beta_{1}^{(k) 2}+\omega^{2} \beta_{2}^{(k) 2}+r_{7}^{(k) 2}\right)}\left(\omega^{2} \beta_{2}^{(k) 2}-\omega^{2} \beta_{1}^{(k) 2}-r_{7}^{(k) 2}\right) \mathrm{e}^{r_{7}^{(k)} x_{3}}\right]\right\} \text {, } \\
& \sigma_{22}^{*}\left(\beta_{1}^{(k)}, \beta_{2}^{(k)}\right)=\sigma_{11}^{*}\left(\beta_{2}^{(k)}, \beta_{1}^{(k)}\right) \\
& \sigma_{33}^{*}=\sum_{k=0}^{\infty} \frac{\alpha \sigma^{0}}{\Delta}\left(\left(\beta_{1}^{(k)}\right)^{2}+\left(\beta_{2}^{(k)}\right)^{2}-\left(\beta_{3}^{(k)}\right)^{2}\right) \mathrm{e}^{i \omega\left(\beta_{1}^{(k)} x_{1}+\beta_{2}^{(k)} x_{2}\right)}\left\{-\left(\left(\beta_{3}^{(k)}\right)^{2}-\left(\beta^{(k)}\right)^{2}\right) e^{i \omega \beta_{3}^{(k)} x_{3}}-\right. \\
& \left.-\frac{\left(\left(\beta_{1}^{(k)}\right)^{2}+\left(\beta_{2}^{(k)}\right)^{2}\right) i}{r_{5}^{(k)}-r_{7}^{(k)}}\left[\left(\omega \beta_{3}^{(k)}+i r_{7}^{(k)}\right) \mathrm{e}^{r_{5}^{(k)} x_{3}}-\left(\omega \beta_{3}^{(k)}+i r_{5}^{(k)}\right) \mathrm{e}^{r_{7}^{(k)} x_{3}}\right]\right\}, \\
& \sigma_{12}^{*}=\sum_{k=0}^{\infty} \frac{\alpha \sigma^{0}}{\Delta} \mathrm{e}^{i \omega\left(\beta_{1}^{(k)} x_{1}+\beta_{2}^{(k)} x_{2}\right)}\left\{-2 \beta_{1}^{(k)} \beta_{2}^{(k)} \beta_{3}^{(k) 2} \mathrm{e}^{i \omega \beta_{3}^{(k)} x_{3}}+\frac{2 i\left(\beta_{1}^{(k) 2}+\beta_{2}^{(k) 2}-\beta_{3}^{(k) 2}\right) \beta_{1}^{(k)} \beta_{2}^{(k)}}{r_{5}^{(k)}-r_{7}^{(k)}} \times\right. \\
& \left.\times\left[\frac{\left(\omega \beta_{3}^{(k)}+i r_{7}^{(k)}\right) r_{5}^{(k) 2}}{\omega^{2} \beta_{1}^{(k) 2}+\omega^{2} \beta_{2}^{(k) 2}+r_{5}^{(k) 2}} \mathrm{e}^{\mathrm{r}_{5}^{(k)} x_{3}}-\frac{\left(\omega \beta_{3}^{(k)}+i r_{5}^{(k)}\right) r_{7}^{(k) 2}}{\omega^{2} \beta_{1}^{(k) 2}+\omega^{2} \beta_{2}^{(k) 2}+r_{7}^{(k) 2}} \mathrm{e}^{r_{7}^{(k)} x_{3}}\right]\right\} \\
& \sigma_{13}^{*}=\sum_{k=0}^{\infty} \frac{\alpha \sigma^{0}}{\Delta}\left(\beta_{1}^{(k) 2}+\beta_{2}^{(k) 2}-\beta_{3}^{(k) 2}\right) \mathrm{e}^{i \omega\left(\beta_{1}^{(k)} x_{1}+\beta_{2}^{(k)} x_{2}\right)}\left\{-\beta_{1}^{(k)} \beta_{3}^{(k)} e^{i \omega \beta_{3}^{(k)} x_{3}}+\right. \\
& \left.-\frac{\omega \beta_{1}^{(k)}}{r_{5}^{(k)}-r_{7}^{(k)}}\left[\frac{\left(\omega \beta_{3}^{(k)}+i r_{7}^{(k)}\right) r_{5}^{(k)}}{\omega^{2}} \mathrm{e}^{r_{5}^{(k)} x_{3}}-\frac{\left(\omega \beta_{3}^{(k)}+i r_{5}^{(k)}\right) r_{7}^{(k)}}{\omega^{2}} \mathrm{e}^{r_{7}^{(k)} x_{3}}\right]\right\} \text {, } \\
& \sigma_{23}^{*}\left(\beta_{1}^{(k)}, \beta_{2}^{(k)}\right)=\sigma_{13}^{*}\left(\beta_{2}^{(k)}, \beta_{1}^{(k)}\right) .
\end{aligned}
$$

Дальнейшие расчеты произведены при условии, что все величины $\beta_{i}^{(k)}$ равны единице. При этом условии случайное поле $U$, заданное разложением (8), можно считать приближенно изотропным [3].

На основе решения краевой задачи (20) были вычислены дисперсии случайного поля напряжений в любой точке полупространства по формуле:

$$
D_{i j}=\left\langle\left|\sigma_{i j}^{*}\right|^{2}\right\rangle \text {. }
$$

Они будут являться функциями $\omega x_{3}$ ( $\omega$ - частота флуктуаций микронеоднородности). В силу громоздкости выражения для дисперсий здесь не выписаны.

Для оценки напряженного состояния полупространства были вычислены нормированные дисперсии

$$
D_{i j}^{0}=\frac{D_{i j}\left(x_{3}\right)}{D_{i j}(\infty)} .
$$

Графики нормированных дисперсий при различных значениях степени нелинейности материала, представлены на рис. 1 и 2.

Поле напряжений в некотором пограничном слое является статистически неоднородным вдоль оси $x_{3}$, т.е. в направлении, нормальном к границе полупространства. Вне этого слоя поле напряжений является однородным, причем оно совпадает с полем напряжений для неограниченной среды.

Из рис. 1 видно, что при $\omega x_{3} \geq 5$ ошибка от замены нормированных дисперсий единицей не превосходит 5\%. Поэтому можно считать, что зона пограничного слоя имеет ширину поряд- 
ка $4,5 / \omega$. С ростом порядка нелинейности материала $n$ зона пограничного слоя увеличивается. Например, как видно из рис. 2 , при $n=7$ она имеет ширину порядка $6 / \omega$.

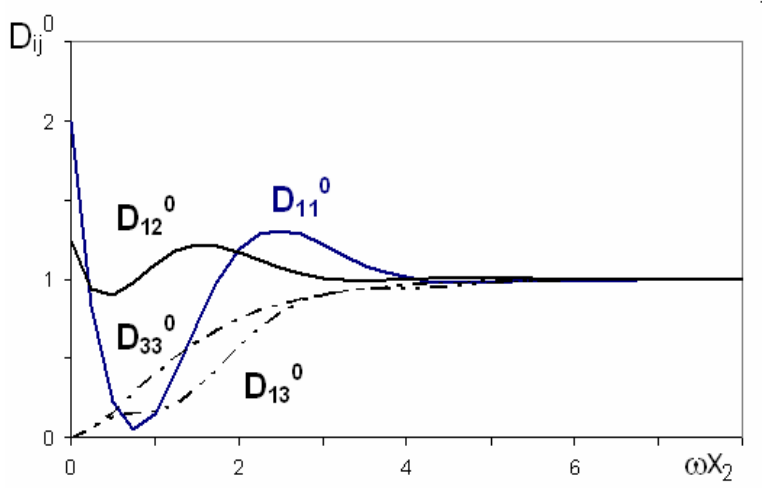

Р и с. 1. Графики нормированных дисперсии при

$$
n=3
$$

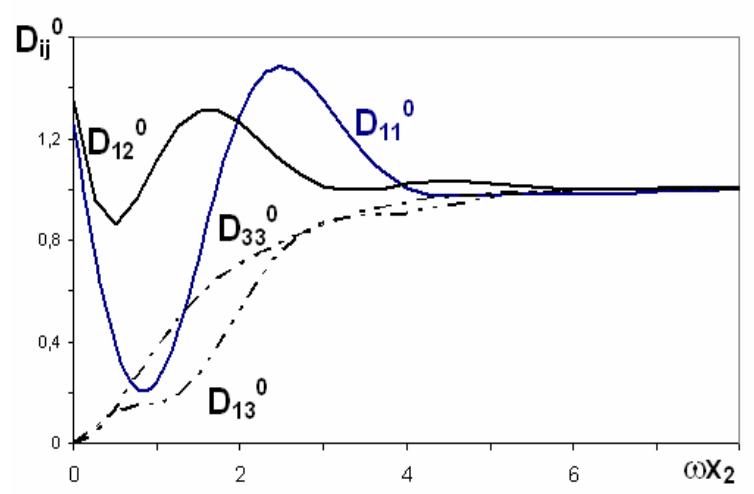

Р и с. 2. Графики нормированных дисперсии при $n=7$

Также была посчитана концентрация напряжений $\sigma_{11}^{*}$ и $\sigma_{22}^{*}$, возникающая на границе поверхности $x_{3}=0$ в зависимости от степени нелинейности материала. Она вычисляется по формуле

$$
\rho=\frac{\sqrt{D_{11}(0)}}{\sqrt{D_{11}(\infty)}},
$$

a ее значения в зависимости от показателя нелинейности установившейся ползучести приведены в таблице

\section{Концентрация напряжений $\rho$ на границе поверхности}

\begin{tabular}{|c|c|c|c|c|}
\hline$n$ & 3 & 5 & 7 & 9 \\
\hline$\rho$ & 1,414 & 1,204 & 1,121 & 1,080 \\
\hline
\end{tabular}

Как следует из таблицы, величина $\rho$ изменяется в пределах от 1,08 до 1,41 . При этом с увеличением $n$ концентрация напряжений уменьшается. Для сравнения, значение этой величины для упругого полупространства при коэффициенте Пуассона $v=0,25$ равно 1,05 [5].

Таким образом, в поверхностном слое флуктуации напряжения достигают заметных величин, которые могут быть значительно больше, чем для глубинных слоев. Следовательно, флуктуации напряжения в пограничном слое играют существенную роль при решении вопроса о надежности конструкций. Неучет краевых эффектов может привести к необоснованному завышению оценок работоспособности элементов конструкции, используемых в производстве.

\section{БИБЛИОГРАФИЧЕСКИЙ СПИСОК}

1. Попов Н. Н., Самарин Ю. П. Пространственная задача стационарной ползучести стохастически неоднородной среды // ПМТФ, 1985. № 2. С. 150-155.

2. Попов Н. Н., Самарин Ю. П. Исследование полей напряжений вблизи границы стохастически неоднородной полуплоскости при ползучести // ПМТФ, 1988. № 1. С. 159-164.

3. Попов Н. Н., Коваленко Л. В. Поле напряжений на границе стохастически неоднородной полуплоскости при ползучести // Вестн. Сам. гос. техн. ун-т. Сер.: Физ.-мат. науки, 2006. Вып. 42. С. 61-66.

4. Ломакин B. А. Статистические задачи механики твердых деформируемых тел. М.: Наука, 1970. 137c.

5. Подалков B. B., Романов B. А. Концентрация напряжений на границе микронеоднородного упругого полупространства // ПММ, 1978. Т. 42. Вып.3. С. 540-545. 